\title{
SEGREGACIÓN ESCOLAR POR ORIGEN NACIONAL EN ESPAÑA ${ }^{1}$ SCHOOL SEGREGATION BY NATIONAL ORIGIN IN SPAIN
}

\author{
F. Javier Murillo \\ Universidad Autónoma de Madrid, España \\ javier.murillo@uam.es \\ Cynthia Martínez-Garrido \\ Universidad Autónoma de Madrid, España \\ cynthia.martinez@uam.es \\ Guillermina Belavi \\ Universidad Autónoma de Madrid, España \\ guillermina.belavi@uam.es
}

\begin{abstract}
Cómo citar / Citation
Murillo, F. Javier; Martínez-Garrido, Cynthia y Belavi, Guillermina (2017). "Segregación escolar por origen nacional en España". OBETS. Revista de Ciencias Sociales, 12(2): pp-pp. 395-423. doi:10.14198/OBETS2017.12.2.04

\section{Resumen}

Esta investigación busca estimar la magnitud de la segregación escolar por origen nacional en España y sus Comunidades Autónomas en Educación Secundaria Obligatoria y contrastar estos resultados con los de los países de la Unión Europea. Para ello, se realiza una explotación especial de los datos PISA2015 estimando el índice de Gorard. Los resultados indican que la magnitud de la segregación escolar por origen nacional en España es 0,45 (por debajo de la media europea). Sin embargo, los valores de la segregación neta reflejan una imagen menos optimista, de ser el tercer país con menos segregación de la Unión Europea, pasa a ser el séptimo.
\end{abstract}

\footnotetext{
${ }^{1}$ Este artículo ha sido desarrollado en el marco del Proyecto I+D+i de Excelencia "Escuelas en contextos socio-económicamente desafiantes: Una aproximación desde la Educación para la Justicia Social" Ref: EDU2014-56118-P. Financiado con fondos del Ministerio de Economía y Competitividad-Gobierno de España.
} 
Palabras Clave: Segregación escolar, Nativos, Inmigrante, España.

\section{Abstract}

The aims of this research are to estimate the magnitude of school segregation by national origin in compulsory secondary education in Spain and its Autonomous Communities, and to compare with the results from other countries in the European Union. In order to achieve those aims, we used the PISA-2015 database and estimate the Gorard index. Our results indicate that the magnitude of school segregation by national origin in Spain is 0.45 (below the European average). However, net segregation values reflect a less optimistic image for Spain, from the third country with the least segregation of the European Union, becomes the seventh.

Keywords: School segregation, Natives, Immigrant, Spain.

\section{Extended Abstract}

An educational system that segregates students according to their personal or social characteristics reproduces inequalities and perpetuates social injustice. Only if we know about school segregation, we will be able to work against it. This article focuses on school segregation by national origin of the students, that is, the phenomenon whereby exists a differential concentration of students in some schools or in others depending on whether they were born or not in the country in which they reside and study.

In recent years, there has been a steady increase in immigration in many European countries, including Spain. International research cautions about a high degree of global segregation for immigrant students that is increasing over the time (Dronkers \& Levels, 2008, Entorf \& Lauk, 2008, Schnepf, 2006). In Spain, the subject has been studied deeply, but only in the case of some regions, especially Catalonia, where has been found a wide variability in school segregation according to different municipalities (Baetbó, 2007, Benito \& González, 2008, Bonal 2012, Sánchez Hugalde, 2007, Sindic de Greuges, 2008, Valiente, 2008).

Given the seriousness of the European situation and the lack of differentiated information about segregation by origin in Spain and its Autonomous Communities, the present research proposes: i) to estimate the magnitude of school segregation by national origin in Spain in Compulsory Secondary Education differentiating by Communities, and ii) to estimate the school segregation in the countries of the European Union to contrast the results obtained in Spain. METHODS

To achieve our aims, we carry out a secondary exploitation of the available data from the PISA-2015 international evaluation.

We use two different samples. The first one corresponds to the database of Spain, given by the total state's information and by that of each Autonomous Communities. This sample has the information of 39,066 students of 15 years old enrolled in 1,177 educational centers. The second sample corresponds to data from the 25 European Union countries that participated in PISA-2015 apart from Spain, and includes 164,938 students enrolled in 6,126 centers, with an average of 6,598 students per country. 
The variable criterion used in this study is the national origin of the student that PISA describes with three alternatives: native, for cases in which both the student and his parents were born in the reference country, immigrant lst generation, for cases in which the student was born in a different country, and immigrant 2nd generation, for cases in which the student was born in the country of reference, but his two parents were born in a different one. In the sample of Spain, 9.8\% are immigrants of 1st generation, and 1.8\% of 2nd generation. In the sample of countries of the European Union, $6.3 \%$ of the students are 1st generation immigrants and $4.3 \%$ 2nd generation. The cases that had no information of the variable criterion were eliminated from the database.

The segregation was estimated using the Gorard index, which considers the magnitude of segregation through the calculation of the proportion of subjects in the minority group who should change their organizational unit to reach a homogeneous distribution among the different establishments. For doing so, it estimates the level of segregation by considering the difference between the proportion of the minority group and the proportion of all members of the group.

RESULTS

Segregation in Spain and in its Autonomous Communities

The magnitude of school segregation by national origin in Compulsory Secondary Education in Spain, measured by the Gorard index, is 0.45 when only the first-generation immigrants are considered, and 0.44 if both the 1st and 2 nd generation are considered. Taking into account only the 1st generation of immigrant students, it is possible to establish four large groups according to the situation of each Autonomy: a) Communities with hypersegregation (more than 0.5 in the Gorard index): Extremadura (0.63) and the Basque Country $(0,54)$. B) Communities with high segregation (between 0.4 and 0.5): Andalusia (0.45) and Galicia (0.44). C) Communities with moderate segregation (between 0,3 and 0,4$)$ : Navarre $(0,40)$, Catalonia $(0,40)$, Asturias $(0,38)$, Cantabria $(0,38),(0,38)$, Castile and Leon $(0,37)$, CastillaLa Mancha $(0,34)$, Balearic Islands $(0,34)$, Community of Madrid $(0,31)$, Region of Murcia 31) and Aragon (0.30), and d) Communities with low segregation (less than 0.3): La Rioja (0.26).

School segregation by origin in the context of the European Union

The differential study of lst generation immigrant students indicates that school segregation by origin in Spain, although high, is below the average of the countries of the European Union. Considering the 23 countries that has been studied, Spain is only above Luxembourg (0.34) and Ireland (0.36) and far below Germany, with 0.64 , the Netherlands with 0.58 , or even France, with a 0,57 .

The situation is different if both first and second-generation immigrant students are considered. In this case, Spain's segregation (0.44) is closer to the European Union average (0.50), and is higher than Portugal (0.43), Italy (0.42), Belgium $(0,42)$, Greece $(0,41)$, Sweden $(0,40)$, Germany $(0,39)$ or Ireland $(0,30)$; although it is still lower than others such as France (0.45), Netherlands (0.48) or Finland (0.50). 
Relationship between segregation and percentage of immigrants According to our data, the correlation between the level of segregation in each Spanish Autonomous Community or EU country and the percentage of immigrants gives high and negative values ( $r x y=-0.686$ and $r x y=-0.598)$. In other words, the more immigrant students enrolled in a community or a country, the lower the rate of segregation they have. Considering this, if we estimate segregation by "discounting" the influence of the number of immigrants enrolled, the results are different: Four regions have a much higher segregation than expected: Catalonia, the Canary Islands, Basque Country, and Estremadura (residuals greater than 1) and three communities have a much smaller segregation than predicted (residues less than -1): Castilla-La Mancha, Asturias, and Castile and Leon.

Among the EU countries the most striking change is that of Luxembourg, which becomes one of the countries with the greatest relative segregation; Germany' rate of school segregation is also worse when it is considered in relative terms. However, Slovenia, and the Netherlands are the most segregated, considering both gross and net rates. The countries with lower segregation's rates are the ones of the Western Europe, especially Ireland, Italy, Portugal and Finland.

This new vision of net segregation offers a less optimistic image for Spain, since from being the third country with the least segregation by origin now becomes the seventh, reaching a higher segregation rate than the United Kingdom.

CONCLUSIONS

This research has shown, with current and statistically representative data, that school segregation by national origin is a severe problem in Spain, even when most European Union countries have higher segregation rates if only the first-generation immigrants are considered. One of the most interesting points in the research is the relationship between school segregation by national origin and the percentage of immigrants in the education system, and its consideration in the calculation of the segregation rates of the Autonomous Communities and European countries.

Variables such as the different processes of decentralization followed by each Community and the ideological orientation of the regional government are factors that affect the management and direction of education and have profound consequences on school segregation. This work is pioneer in making visible these consequences since it presents the situation of each Autonomous Community regarding the school segregation by national origin of the students. The data are persistent in showing that Catalonia, the Canary Islands, Basque Country and Estremadura have still much to do.

The study made use of current and reliable data, and a representative sample for each Autonomous Community. The PISA-2015 database provides latest information and a highly significant sample. These results are added to the findings of national and international research of school segregation and reinforce the need to address complementary studies and design educational policies that take on the problem and pose lasting solutions. 
Only by knowing in depth the problem of school segregation in Spain will be possible to take effective measures to combat it. The present article intends to have made a small contribution in this sense.

\section{INTRODUCCIÓN}

¿Puede la escuela contribuir en la construcción de una sociedad más justa y equitativa? Queremos creer que sí. Sin embargo, una escuela a la que se niegue la oportunidad de educar en convivencia a personas de diferentes procedencias, culturas y características, difícilmente podrá contribuir a ello. Al contrario, una escuela homogénea, que no represente la variabilidad social, seguramente será un potente medio de reproducción de las desigualdades. Un sistema educativo que segrega a los estudiantes en unas escuelas u otras simplemente por sus características personales o sociales no garantiza una real igualdad de oportunidades para todos y todas.

El estudio de la segregación escolar se nos presenta, así, como un medio imprescindible para conocer y, con ello, cambiar una situación directamente relacionada con la equidad de los sistemas educativos y su posibilidad de ser un medio de transformación social. Uno de los tipos de segregación escolar es la segregación por origen nacional, que considera como criterio el origen nacional del estudiante, es decir, toma en cuenta si el estudiante ha nacido o no en el país donde reside y estudia. De esta manera, se entiende la segregación como el fenómeno por el cual los estudiantes inmigrantes se concentran en unas escuelas y no en otras, generando guetos y negando la posibilidad de que el centro sea un espacio de encuentro e interrelación entre culturas.

En general y al menos durante los primeros años de residencia, los inmigrantes se encuentran en condiciones de inferioridad respecto al resto de ciudadanos en aspectos como el trabajo, la vivienda, la cultura, y no sólo como individuos, también como grupo social (marginación, xenofobia, dificultades para la participación, etc.) (García Castaño y Olmos Alcaraz, 2012). El grueso de la inmigración que recibe España proviene de países más pobres, se trata de personas que abandonan su país de origen atraídas por mejores condiciones de vida y en busca de nuevas oportunidades de trabajo (Aja, 2000). De modo que conocer y tratar la segregación escolar contribuye a evitar que las dificultades culturales se retroalimenten con dificultades socio-económicas propias de los barrios en los que se asienta la población inmigrante.

La tendencia a la agrupación es una característica general de la inmigración (Aja, 2000; Garreta, 2011). Los nuevos residentes tienden a concentrarse en determinadas ciudades, incluso en determinados barrios, principalmente debido a razones económicas, pero también por la proximidad con compatriotas o 
familiares. De esta manera, existe una tendencia "natural" a la concentración de los estudiantes inmigrantes e hijos de inmigrantes en determinados centros. Esto dificulta al sistema educativo revertir una tendencia a la segregación. Pero, además, la literatura llama la atención sobre una causa "artificial" de segregación, pues las familias autóctonas retiran a sus hijos de los centros en los que predominan los estudiantes de origen extranjero (Frankenberg, 2013). Se inicia así un proceso de segregación desde la escuela que puede significar la guetización de algunos centros escolares.

Sabemos que actualmente en España están escolarizados en enseñanzas no universitarias un total de 724.635 alumnos inmigrantes. En Educación Secundaria Obligatoria, 179.973 de los 1.840 .748 estudiantes matriculados no han nacido en España, lo que implica que un 9,78\% de los estudiantes de ese nivel educativo son inmigrantes de $1^{a}$ generación (MECD, 2016). Sin embargo, desconocemos cómo están distribuidos esos estudiantes en los centros. Aunque podemos intuir que algunas escuelas concentran a la mayoría de ellos, existen muy pocos estudios al respecto y menos aún en el conjunto del Estado español. Tampoco sabemos si hay diferencias por Comunidades o si esa situación es mayor o menor que la segregación de otros países de nuestro entorno. La búsqueda de una respuesta a los anteriores interrogantes orienta esta investigación.

Formalmente, esta investigación busca alcanzar dos objetivos. Por un lado, estimar la magnitud de la segregación escolar por origen en España en Educación Secundaria Obligatoria diferenciando los resultados por Comunidades y, por otro, estimar la segregación escolar en los países de la Unión Europea para contrastar los resultados obtenidos en España. Para ello, se hace una explotación especial de los últimos datos disponibles de la evaluación internacional PISA, correspondientes a 2015 y que fueron liberados en diciembre de 2016 (OCDE, 2016).

\section{REVISIÓN DE LA LITERATURA}

Hace poco se cumplieron 60 años de uno de los hitos que marcaron la historia de la escuela en los Estados Unidos y que constituye uno de los logros más importantes del Movimiento por los Derechos Civiles americanos. Hablamos de la sentencia de la corte suprema de los Estados Unidos de 17 de mayo de 1954 llamada Brown v. Consejo de Educación de Topeka, por la que se declaró inconstitucional la separación de estudiantes en función de su raza en distintas escuelas por atentar al principio de igualdad de oportunidades educativas de todas las personas. La verificación del fin de la segregación, en primer lugar de carácter étnico-racial, posteriormente por otras razones, generó a finales de los años 50 en Estados Unidos una fructífera línea de investigación educa- 
tiva que busca conocer la magnitud de la segregación escolar, pero también sus causas y sus efectos educativos y sociales.

Se entiende por segregación escolar la desigual distribución de los estudiantes en las escuelas en función de sus características personales o sociales (Capellán de Toro et al., 2013). Es decir, el fenómeno por el cual los estudiantes con determinadas características o condiciones se concentran en algunas escuelas y no en otras; puede tratarse de estudiantes pertenecientes a un grupo cultural minoritario, estudiantes con familias de determinado nivel socioeconómico, o estudiantes que ellos ( $1^{\circ}$ generación) o sus padres $\left(2^{\circ}\right.$ generación) no han nacido en el país donde residen y estudian. Este último caso es el que nos interesa en esta investigación, es la llamada segregación escolar por origen nacional del alumnado. En otras publicaciones (p. ej., Murillo y MartínezGarrido, 2018), hemos abordado la segregación de carácter socioeconómico en España.

Como hemos señalado la investigación sobre segregación escolar nace a finales de la década de los años 50 y principios de los años 60. Inicialmente se centró en la segregación de los estudiantes en función de su pertenencia a un grupo étnico determinado (caucásicos, afroamericanos, latinos...). En los últimos años, diversas investigaciones han evidenciado que la segregación escolar étnico-racial está disminuyendo, por lo que los trabajos sobre esta temática también decrecen en número (Stroub y Richards, 2013). Sin embargo, aumenta la preocupación y el interés por la segregación escolar generada por el origen nacional del alumnado. Este hecho es especialmente acusado en Europa donde se están mostrando evidencias, a veces dolorosas, del fracaso de las políticas de integración o asimilación de las personas inmigrantes (El-Habib et al., 2016; Entorf y Lauk, 2008). Y, una vez más, la mirada se vuelve a la escuela.

Efectivamente, el rápido incremento de inmigrantes en algunas partes del mundo ha suscitado el interés de la comunidad educativa por conocer el progreso de estos estudiantes, tanto en los países considerados clásicos receptores (Australia o Estados Unidos), como en aquellos donde la llegada de inmigrantes ha aumentado relativamente en los últimos años (por ejemplo, España o Grecia) (Capellán de Toro et al., 2013; Palaiologou, 2007; Terren y Carrasco, 2007). Los resultados de estas investigaciones evidencian un alto grado de segregación global para los estudiantes inmigrantes y, lo que es más preocupante, su aumento en el tiempo, tanto entre quiénes son inmigrantes de $1^{\mathrm{a}}$ como de $2^{a}$ generación (Noguera, Pierce y Ahram, 2015).

De entre las investigaciones realizadas sobre segregación escolar por origen nacional podemos distinguir los estudios internacionales, que usan grandes bases de datos -por ejemplo, Schnepf (2006, 2007), Dronkers y Levels 
(2008), Entorf y Lauk (2008), Schneeweis (2011)-, y los que analizan el estado de la segregación escolar en algunos países -por ejemplo, en Reino Unido (Burgess et al., 2004; Simpson, 2007)-. De entre los primeros, el trabajo elaborado por Schneeweis (2011) destaca por ser quizá el más ambicioso de todos los realizados hasta la fecha en el estudio de la segregación escolar por origen nacional. La autora estudia un total de 167 países de los cinco continentes a partir de los datos de PISA-2009 y TIMMS. Aunque en los resultados no se aportan datos desagregados de cada país, el estudio indica que para que el reparto de los estudiantes nativos e inmigrantes fuera homogéneo entre las escuelas, sería necesario que el 38,6\% de los estudiantes inmigrantes cambiara de centro escolar (valor del índice de Disimilitud).

Otro estudio realizado con datos de varios países es el desarrollado por Schnepf (2006). La autora busca conocer el estado de la segregación escolar de los estudiantes inmigrantes en la OCDE e identificar la relación entre el número de inmigrantes en cada escuela y el nivel de segregación alcanzado. De acuerdo con los resultados de la investigación, existe una relación negativa entre el número de inmigrantes y el índice de segregación; de manera que las escuelas con mayor proporción de estudiantes inmigrantes son las que alcanzan niveles más bajos de segregación. Por ejemplo, Australia y Suiza destacan por tener mayor cantidad de población inmigrante en sus escuelas (23\% y 19\%, respectivamente) y su índice de segregación es 0,40 y 0,48 , respectivamente. Al contrario, Reino Unido y Estados Unidos, que cuentan con la menor proporción de inmigrantes en sus escuelas (9\%) alcanzan un nivel de segregación escolar por origen de 0,61 y 0,65, respectivamente. Estos resultados son coherentes con los aportados por Entorf y Lauk (2008). Según estos autores, en la República Checa y en Hungría las escuelas cuentan con un 1\% de estudiantes inmigrantes y los valores de su índice de segregación son los más altos de entre los países estudiados ( 0,82 y 0,61 , respectivamente), les sigue Noruega donde sólo el 4\% de los estudiantes en las escuelas es inmigrante o Austria donde lo es el 9\%, ambas con un índice de segregación de 0,58. En el extremo contrario, Australia cuenta con el mayor porcentaje de estudiantes inmigrantes en sus escuelas $(22 \%)$ y obtiene el menor índice de segregación en el estudio $(0,48)$ (valores medidos a través de índice de Disimilitud).

También es interesante el trabajo realizado por Dronkers y Levels (2008). Estos investigadores holandeses, partiendo de los datos de PISA-2003, obtienen que el valor promedio de la segregación escolar por origen para los estudiantes inmigrantes de $2^{a}$ generación es menor que para los estudiantes inmigrantes de $1^{a}$ generación ( $8,8 \%$ frente a $10,2 \%$, respectivamente), pero esta tendencia no ocurre en todos los países. Según los autores, en Australia (9,9\%; 
$8,1 \%)$, Dinamarca $(5,9 \% ; 2,5 \%)$, Alemania $(9,8 \% ; 7,2 \%)$, y Letonia $(10,9 \%$; $3,2 \%)$ son los estudiantes inmigrantes de $2^{a}$ generación los que experimentan mayores niveles de segregación escolar en comparación con los estudiantes inmigrantes de $1^{a}$ generación (valores medidos a través de la desviación típica del valor promedio de inmigrantes en cada escuela).

Entre las investigaciones que analizan el nivel de segregación escolar por origen nacional en un país determinado destacan los trabajos realizados por Burgess y colaboradores (2004) y Simpson (2007) para Reino Unido. De un lado, Burgess y colaboradores (2004) indican que el valor promedio de la segregación escolar por origen en el Reino Unido es 0,633 (valor medido a través del índice de Disimilitud). De otro, Simpson (2007) señala que en Inglaterra y Gales los estudiantes inmigrantes más segregados son los paquistaníes $(0,75)$ y bangladesíes $(0,74)$, seguidos de los africanos $(0,71)$, caribeños $(0,68)$, indios $(0,65)$ y chinos $(0,42)$ (valores medidos a través del índice de Disimilitud).

En España, no es hasta principios de los años 2000 cuando se realizan los primeros estudios sobre segregación escolar por origen, motivados según Castaño, Gómez y Bouachra (2008) por el interés por conocer la eficacia de las medidas tomadas para la integración del alumnado extranjero en el país. Así, ya contamos con buenas investigaciones realizadas con datos del conjunto de Estado y sus Comunidades Autónomas (Gallego y Subirats, 2011), pero especialmente trabajos que se centran en analizar la segregación en Cataluña (Alegre, Benito y González-Baetbó, 2008; Benito y González-Baetbó, 2007; Bonal 2012; Sánchez Hugalde, 2007; Sindic, 2008; Valiente, 2008).

La investigación evidencia grandes diferencias en el nivel de segregación por origen entre municipios de una misma comunidad. De acuerdo con Sánchez Hugalde (2007) algunos municipios cuentan con niveles bajos de segregación escolar por origen $(0,19$ en el Valle de Arán) frente a otros, como Barcelona, que alcanzan niveles de híper-segregación $(0,62)$ (valores medidos a través del índice de Disimilitud). Cifras que son coherentes con las aportadas por Benito y González-Baetbó (2007), quienes señalan diferencias de hasta 0,25 puntos entre los niveles de segregación escolar entre municipios para inmigrantes de $2^{a}$ generación, y de hasta 0,41 puntos para inmigrantes de $1^{a}$ generación. De acuerdo con sus resultados, el nivel de segregación escolar de los estudiantes de $1^{a}$ generación es mayor al de los estudiantes de $2^{a}$ generación, 0,4 frente a 0,36 (valores medidos a través del índice de Gorard).

Por su parte, Alegre, Benito y González-Baetbó (2008) y Bonal (2012) estudian la variación del valor de la segregación escolar por origen en función de la etapa educativa y de la política establecida para la elección del centro. Los resultados indican que el nivel de segregación escolar por origen en las escuelas 
de Educación Secundaria es ligeramente menor que en las de Educación Primaria, 0,12 frente a 0,19, respectivamente (Bonal, 2012) (valor medido a través del índice de Hutchens). No ocurre lo mismo si se estudia la distribución de los estudiantes inmigrantes entre las escuelas públicas y privadas. Según Gallego y Subirats (2011) las escuelas de Educación Primaria son más equitativas $(2,78)$ que las escuelas de Educación Secundaria $(2,10)$ (valores medidos a través del índice de Equidad). Por otro lado, los municipios que son considerados por la administración educativa como zona única para la elección de centro escolar por parte de las familias tienen un nivel de segregación menor $(0,32)$ que en aquellos considerados área-escuela $(0,46)$ o zona múltiple $(0,42)$ (Alegre, Benito y González-Baetbó (2008) (valores medidos a través del índice de Gorard).

Por último, destacan el trabajo realizado por Valiente (2008) y el auspiciado por el Síndic de Greuges en Cataluña (Sindic, 2008) acerca de la evolución de la segregación escolar por origen. Por un lado, el informe del Síndic de Greuges de 2008 estudia la evolución del nivel de segregación escolar por origen en Cataluña -desde finales de los 90 hasta el año de publicación del trabajo-y su comparativa en función del nivel educativo: Primaria y Secundaria. Los resultados indican que la segregación escolar por origen puede organizarse en tres períodos: (i) finales de la década de los años 90, cuando había poca intensidad de flujos migratorios, los niveles de segregación eran valores altos (0,55 en Primaria, y 0,47 en Secundaria). (ii) Entre los años 2000 a 2004, hubo una etapa de decrecimiento del nivel de segregación (0,45 en Primaria y 0,37 en Secundaria); y (iii) durante los años 2005 y 2006 los niveles de segregación escolar por origen nacional se mantienen constantes, alcanzando valores moderados (0,46 en Primaria y 0,35 en Secundaria) (valores obtenidos a través del índice de Disimilitud). Tendencia que confirma la investigación elaborada por Valiente (2008) al indicar un descenso de 9 puntos porcentuales en el nivel de la segregación desde 2001 hasta 2006, de 0,55 hasta 0,46 respectivamente (valores medidos a través del índice de Disimilitud). Sin embargo, durante ese mismo período, el aislamiento del alumnado inmigrante en Cataluña creció de un 0,07 a un 0,14 (valores medidos a través del índice de Aislamiento).

Como hemos reflejado, la investigación realizada en España sobre segregación escolar por origen se ha realizado mayoritariamente en Cataluña. Sus resultados destacan las grandes diferencias del nivel de segregación por origen entre unas escuelas y otras, que la segregación es más acusada entre los estudiantes inmigrantes de $1^{\text {a }}$ generación y que, al menos para esta Comunidad, 
actualmente el nivel de segregación por origen en las escuelas parece estar disminuyendo. Resulta por tanto necesario identificar si estos resultados se cumplen para el conjunto del estado español. Por ello, la presente investigación persigue dos objetivos: i) estimar la magnitud de la segregación escolar por origen nacional en España en Educación Secundaria Obligatoria diferenciando por Comunidades, y ii) estimar la segregación escolar en los países de la Unión Europea para contrastar los resultados obtenidos en España.

\section{MÉTODO}

Para alcanzar dichos objetivos se lleva a cabo una explotación secundaria de los datos aportados por las diferentes Comunidades Autónomas españolas en la evaluación internacional PISA realizada en 2015 que se liberaron en diciembre de 2016.

Para cumplir los objetivos, utilizamos dos muestras diferentes. Por un lado, para conocer la magnitud de la segregación en España y en las diferentes Comunidades Autónomas, se seleccionaron los datos del total estatal y de las distintas Comunidades Autónomas, que en la base de datos de PISA-2015 conforman conjuntos diferentes. De esta forma, esta primera muestra está conformada por 39.066 estudiantes de $4^{a}$ de Educación Secundaria Obligatoria escolarizados en 1.177 centros educativos españoles (tabla 1). De ellos 6.736 formaron parte de la muestra del total estatal y los más de 32.000 restantes de las diferentes Comunidades, con una participación promedio de 1.902 estudiantes por comunidad excepto País Vasco que duplicó la muestra. Para comparar los datos de España con los países de su entorno, se seleccionaron los datos de los 25 países de la Unión Europea que, además de España, participaron en PISA-2015. Ello implica 164.938 estudiantes escolarizados en 6.126 centros, con un promedio de 6.598 estudiantes por país.

La variable criterio utilizada en este estudio es el origen nacional del estudiante, estimado por PISA bajo el nombre de Index Inmigration Status y con tres alternativas: nativo, es decir, que tanto el estudiante como sus padres nacieron en el país en el que estudia, inmigrante de $1^{a}$ generación, que nació en otro país, e inmigrante de $2^{a}$ generación, que nació en el país en el que estudia pero sus dos progenitores nacieron en uno diferente. En la muestra de España, el 9,8\% son inmigrantes de $1^{a}$ generación y el 1,8\% de $2^{a}$ generación. En la muestra de países de la Unión Europea, el 6,3\% de los estudiantes son inmigrantes de $1^{a}$ generación y el $4,3 \%$ de $2^{a}$ generación. Todos aquellos casos en los que no se aportaba información de la variable criterio fueron eliminados de la base de datos. 
Tabla 1. Muestra del estudio y sus características. Número de estudiantes y escuelas, y porcentaje de estudiantes inmigrantes de $1^{\mathrm{a}}$ y $2^{\mathrm{a}}$ generación, por país y Comunidad Autónoma

\begin{tabular}{|c|c|c|c|c|}
\hline & $\begin{array}{l}\mathrm{N}^{\mathrm{a}} \text { Estudiantes } \\
\text { muestra }\end{array}$ & $\mathrm{N}^{\circ}$ Escuelas & $\begin{array}{c}\% \text { inmigrantes } \\
1^{\text {a }} \text { gen }^{*}\end{array}$ & $\begin{array}{c}\% \text { inmigrantes } \\
2^{\mathrm{a}} \text { gen }^{*} \\
\end{array}$ \\
\hline España & 6.736 & 201 & 9,07 & 1,93 \\
\hline Andalucía & 1.813 & 54 & 3,23 & 0,87 \\
\hline Aragón & 1.798 & 53 & 12,45 & 1,41 \\
\hline Ppdo. de Asturias & 1.790 & 54 & 5,79 & 0,72 \\
\hline Illes Balears & 1.797 & 54 & 14,24 & 3,01 \\
\hline Canarias & 1.842 & 54 & 9,60 & 3,35 \\
\hline Cantabria & 1.924 & 56 & 9,00 & 0,50 \\
\hline Castilla y León & 1.858 & 57 & 6,60 & 0,84 \\
\hline Castilla-La Mancha & 1.889 & 55 & 7,63 & 1,34 \\
\hline Cataluña & 1.769 & 52 & 14,81 & 4,03 \\
\hline Extremadura & 1.809 & 53 & 1,74 & 0,38 \\
\hline Galicia & 1.865 & 59 & 5,07 & 1,05 \\
\hline La Rioja & 1.461 & 47 & 15,70 & 1,36 \\
\hline Com. de Madrid & 1.808 & 51 & 14,84 & 4,69 \\
\hline Región de Murcia & 1.796 & 53 & 12,77 & 2,54 \\
\hline C. Foral de Navarra & 1.874 & 52 & 13,10 & 1,06 \\
\hline País Vasco & 3.612 & 119 & 7,75 & 1,04 \\
\hline Comunitat Valenciana & 1.625 & 53 & 12,28 & 2,29 \\
\hline Alemania & 6.504 & 256 & 13,17 & 3,75 \\
\hline Austria & 7.007 & 269 & 12,71 & 7,59 \\
\hline Bélgica & 9.651 & 288 & 9,02 & 8,70 \\
\hline Bulgaria & 5.928 & 180 & 0,55 & 0,49 \\
\hline Croacia & 5.809 & 160 & 9,02 & 1,78 \\
\hline Dinamarca & 7.161 & 333 & 7,90 & 2,77 \\
\hline Eslovaquia & 6.350 & 290 & 0,61 & 0,59 \\
\hline Eslovenia & 6.406 & 333 & 4,49 & 3,32 \\
\hline Estonia & 5.587 & 206 & 9,32 & 0,66 \\
\hline
\end{tabular}




\begin{tabular}{l|r|r|r|r}
\hline & $\begin{array}{c}\mathbf{N}^{\mathrm{a}} \text { Estudiantes } \\
\text { muestra }\end{array}$ & $\mathbf{N}^{\mathrm{o}}$ Escuelas & $\begin{array}{c}\text { \% inmigrantes } \\
\mathbf{1}^{\mathrm{a}} \text { gen }^{*}\end{array}$ & $\begin{array}{c}\text { \% inmigrantes } \\
\mathbf{2}^{\mathrm{a}} \text { gen }^{*}\end{array}$ \\
\hline Finlandia & 5.882 & 168 & 1,79 & 2,19 \\
\hline Francia & 6.108 & 252 & 8,72 & 4,45 \\
\hline Grecia & 5.532 & 211 & 6,97 & 3,78 \\
\hline Hungría & 5.658 & 245 & 1,54 & 1,15 \\
\hline Irlanda & 5.741 & 167 & 3,36 & 11,02 \\
\hline Italia & 11.583 & 474 & 3,15 & 4,83 \\
\hline Lituania & 6.525 & 311 & 1,37 & 0,39 \\
\hline Luxemburgo & 5.299 & 44 & 30,61 & 21,37 \\
\hline Malta & 3.634 & 59 & 1,48 & 3,48 \\
\hline Países Bajos & 5.385 & 187 & 8,58 & 2,16 \\
\hline Polonia & 4.478 & 169 & 0,10 & 0,15 \\
\hline Portugal & 7.325 & 246 & 3,27 & 4,06 \\
\hline Reino Unido & 14.157 & 550 & 7,98 & 8,76 \\
\hline Rep. Checa & 6.894 & 344 & 1,66 & 1,72 \\
\hline Rumanía & 4.876 & 182 & 0,25 & 0,13 \\
\hline Suecia & 5.458 & 202 & 9,80 & 7,56 \\
\hline Total/promedio muestra & 204.004 & 7.303 & 7,75 & 3,24 \\
\hline Nota * Datos & & & & \\
\hline
\end{tabular}

Nota: * Datos ponderados

Fuente: Elaboración propia a partir de datos de PISA-2015

Para alcanzar los objetivos del estudio se utilizó el índice de segregación de Gorard. La razón de esta elección es que, frente a otros índices como el de Disimilitud, el de Aislamiento, o el de Raíz Cuadrada, es invariante para el tamaño del grupo minoritario. Dado que en cada Comunidad Autónoma y país el porcentaje de estudiantes inmigrantes es diferente, resulta la mejor opción. Las características, potencialidades y peculiaridades de cada índice pueden consultarse en Murillo (2016).

El índice de segregación de Gorard, o índice G (Gorard y Taylor, 2003), es una variación del popular índice de Disimilitud (Duncan y Duncan, 1955), pero supera la limitación de estar influido por el tamaño del grupo minoritario. Según el índice G, la magnitud de la segregación se estima a través del cálculo de la proporción de sujetos del grupo minoritario que deberían cambiar de unidad 
organizativa para que exista una distribución homogénea de los mismos entre los distintos establecimientos, estima el nivel de segregación considerando la diferencia entre la proporción del grupo minoritario y la proporción de todos los miembros del grupo. Para el cálculo del índice se ha tenido en cuenta los pesos finales de cada estudiante, que suministra la propia base de datos PISA2015 para cada país.

El procedimiento de cálculo es:

$$
G=\frac{1}{2} \sum_{i=1}^{k}\left|\frac{x_{1 i}}{X_{1}}-\frac{T_{i}}{T}\right|
$$

Donde, para Comunidad Autónoma, $\mathrm{x}_{1 \mathrm{i}}$ representa el número de alumnos del grupo minoritario en la escuela $\mathrm{i}, \mathrm{X}_{1}$ es el número total de estudiantes del grupo minoritario en todas las escuelas de la Comunidad, $\mathrm{T}_{\mathrm{i}}$ es el número total de alumnos en la escuela i, y T, número total de alumnos en cada Comunidad.

Como hemos señalado, el índice $G$ tiene como principal característica ser la única medida de segregación utilizable con más de dos casos que aparece completamente libre de la influencia o los cambios en la población (Gorard y Taylor, 2003). De esta forma, es posible comparar Comunidades o Países con diferente porcentaje de inmigrantes estudiantes.

Se estimará la segregación, por una parte, entendiendo como grupo minoritario los estudiantes inmigrantes de $1^{\text {a }}$ generación, y, por otro, los estudiantes inmigrantes de $1^{a}$ y de $2^{a}$ generación.

\section{RESULTADOS}

En coherencia con los objetivos planteados, se presentan, en primer lugar, los resultados de la estimación de la segregación escolar por origen nacional en las Comunidades Autónomas, para posteriormente comparar los datos de España con los de los otros países de la Unión Europea.

\section{Segregación en España y sus Comunidades Autónomas}

La magnitud de la segregación escolar por origen nacional en Educación Secundaria Obligatoria en España, medido a través del índice de Gorard, es de 0,45 si se consideran solo los inmigrantes de $1^{\text {a }}$ generación, y de 0,44 si se tiene en cuenta tanto a los de $1^{a}$ como a los de $2^{a}$ generación. Esta cifra es muy alta y nos da la imagen de un sistema educativo claramente inequitativo, más cercano a ser un medio de reproducción social que de transformación. 
La organización del sistema educativo español, con un carácter descentralizado en las competencias ejecutivas y en el desarrollo normativo de las leyes estatales, genera importantes diferencias entre las Comunidades Autónomas. En efecto, el alto índice de segregación en España oculta una gran variabilidad entre una Comunidad Autónoma y otra (tabla 2). Así, considerando solo los estudiantes de $1^{a}$ generación, es posible establecer cuatro grandes grupos de Autonomías:

- Comunidades con hipersegregación (más de 0,5 en el índice de Gorard): Extremadura $(0,63)$ y País Vasco $(0,54)$.

Tabla 2. Segregación escolar por origen nacional en España por CCAA. Índice de Gorard

\begin{tabular}{l|c|c}
\cline { 2 - 3 } & $\begin{array}{c}\text { Inmigrantes de } \\
1^{\mathrm{a}} \text { generación }\end{array}$ & $\begin{array}{c}\text { Inmigrantes de } \\
1^{\mathrm{a}} \text { y } 2^{\mathrm{a}} \text { generación }\end{array}$ \\
\hline Andalucía & 0,4488 & 0,4485 \\
\hline Aragón & 0,3019 & 0,2935 \\
\hline Principado de Asturias & 0,3785 & 0,3791 \\
\hline Illes Balears & 0,3432 & 0,3045 \\
\hline Canarias & 0,4931 & 0,4516 \\
\hline Cantabria & 0,3772 & 0,3569 \\
\hline Castilla y León & 0,3721 & 0,3750 \\
\hline Castilla-La Mancha & 0,3439 & 0,3396 \\
\hline Cataluña & 0,3966 & 0,3634 \\
\hline Extremadura & 0,6299 & 0,5680 \\
\hline Galicia & 0,4451 & 0,3999 \\
\hline La Rioja & 0,2562 & 0,2709 \\
\hline Comunidad de Madrid & 0,3081 & 0,2766 \\
\hline Región de Murcia & 0,3076 & 0,3027 \\
\hline Comunidad Foral de Navarra & 0,3985 & 0,3828 \\
\hline País Vasco & 0,5375 & 0,5158 \\
\hline Comunitat Valenciana & 0,3759 & 0,3522 \\
\hline España & 0,4529 & 0,4440 \\
\hline Eny & &
\end{tabular}

Fuente: Elaboración propia a partir de datos de PISA-2015 
- Comunidades con una alta segregación escolar por origen nacional (entre 0,4 y 0,5$)$ : Andalucía $(0,45)$ y Galicia $(0,44)$.

- Comunidades con una segregación moderada (entre 0,3 y 0,4): Comunidad Foral de Navarra $(0,40)$, Cataluña $(0,40)$, Principado de Asturias $(0,38)$, Cantabria $(0,38)$, Comunitat Valenciana $(0,38)$, Castilla y León $(0,37)$, Castilla-La Mancha $(0,34)$, Illes Balears $(0,34)$, Comunidad de Madrid (0,31), Región de Murcia $(0,31)$ y Aragón $(0,30)$.

- Comunidades con una segregación baja (menos de 0,3): La Rioja $(0,26)$.

Con ello se evidencia una gran variabilidad existente entre Comunidades, donde el valor de la segregación llega a duplicarse entre unas y otras Comunidades (gráfico 1). Efectivamente, mientras en Extremadura la segregación alcanza un altísimo 0,63, en La Rioja esta cifra se queda en un escaso 0,26.

Considerando los inmigrantes de $1^{a}$ y $2^{a}$ generación, las cifras son muy parecidas, con una ligera disminución en el índice de segregación en la mayoría de las Comunidades Autónomas. Concretamente solo hay dos Comunidades en las que aumenta la segregación cuando se incluye a los adolescentes cuyos padres

Gráfico 1. Segregación escolar por origen nacional en España. Índice de Gorard para estudiantes inmigrantes de $1^{\text {a }}$ generación y de $1^{\text {a }}$ y $2^{\text {a }}$ generación en cada Comunidad Autónoma

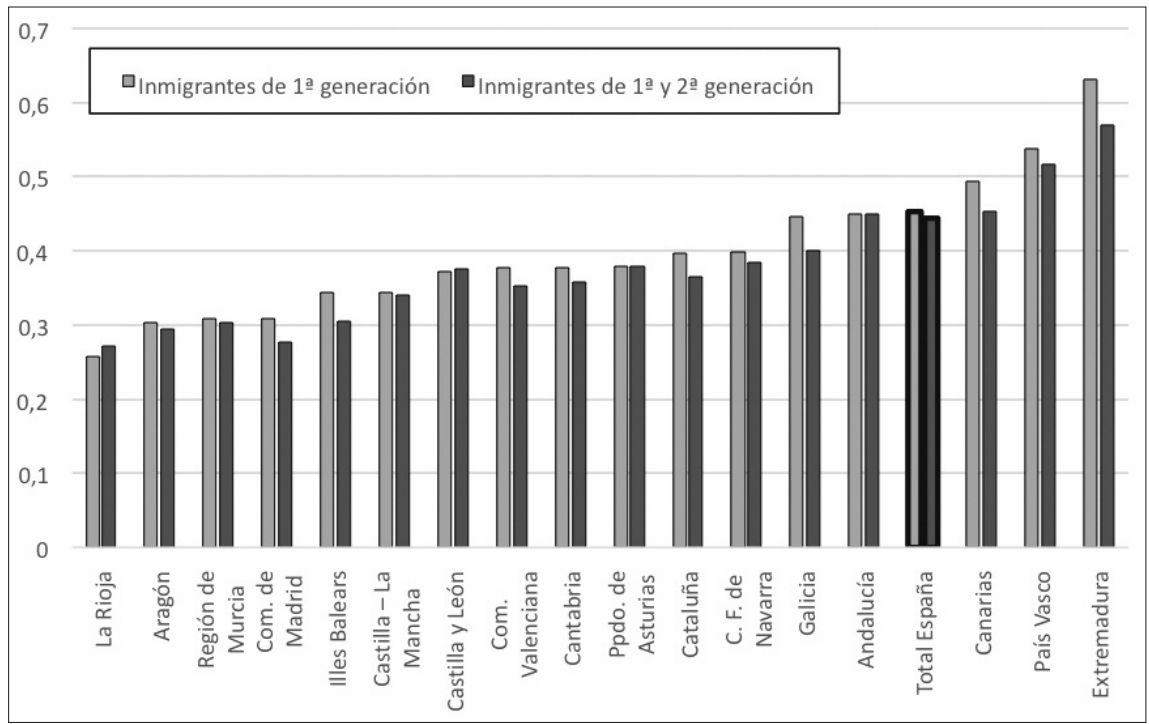

Nota: Comunidades ordenadas en función de la segregación promedio Fuente: Elaboración propia a partir de datos PISA-2015 
no han nacido en España: Asturias y la Rioja. En el primer caso, la diferencia es inapreciable y en el segundo se incrementa de 0,256 a 0,271.

\section{Segregación escolar por origen en el contexto de la Unión Europea}

El análisis de la segregación escolar por origen nacional en los países de la Unión Europea puede ayudar a situar a España en su contexto. En la tabla 3 se muestran los resultados de la estimación de la magnitud de la segregación escolar por origen en los países de la Unión Europea, tanto para estudiantes de $1^{a}$ generación como de $1^{a}$ y $2^{a}$ generación utilizando, como en el caso anterior, el índice de Gorard.

Comenzando por el estudio de la segregación de estudiantes inmigrantes de $1^{a}$ generación (tabla 3 , gráfico 2), una reflexión inicial que merece la pena destacar es que, aun siendo alta la segregación escolar por origen en España, está claramente por debajo de la media de los países de la Unión Europea. De los 23 países estudiados solo está por encima de Luxemburgo $(0,34)$ e Irlanda $(0,36)$ y muy por debajo de países como Alemania, con un 0,64, Países Bajos, con 0,58 , o Francia, con un 0,57. De manera que la falencia del sistema educativo español a la hora de evitar la segregación de estudiantes inmigrantes es común al resto de los países europeos.

La mirada detallada de la segregación en los diferentes países apunta a la existencia de dos grandes bloques de países: los que se encuentran por encima y por debajo del promedio de la Unión Europea en segregación escolar por origen nacional. El primer grupo está conformado casi en exclusiva por países del este europeo y tiene a Rumanía, Polonia y Lituania al frente con un índice de más de 0,9. La gran excepción a esta norma es Países Bajos, que con su 0,63 supera ligeramente el promedio de la Unión Europea $(0,62)$ y le convierte en el país claramente más segregador de la Europa occidental. Seguramente su política de cuasi-mercado escolar no es ajena a esta situación.

En el extremo contrario se encuentran países como Luxemburgo e Irlanda con cifras inferiores a 0,35 que, sin que puedan considerarse bajas, muestran otra forma de afrontar la escolarización de los adolescentes inmigrantes. De este segundo bloque por debajo del promedio de la Unión Europea destacan Eslovenia, con un 0,56 y situada entre Finlandia y Alemania, y Croacia, con un 0,57 ligeramente inferior al promedio de la Unión Europea. Estos dos países, procedentes de la Federación Yugoslava, se encuentran a caballo entre las dos grandes regiones de Europa.

La situación es bastante diferente si consideramos en el análisis tanto a los estudiantes inmigrantes de $1^{a}$ como de $2^{a}$ generación. En este caso la segregación de España $(0,44)$ se acerca más al promedio de la Unión Europa $(0,50)$ y 
Tabla 3. Segregación escolar por origen nacional en los países de la Unión Europea. Índice de Gorard

\begin{tabular}{l|c|c}
\cline { 2 - 3 } & $\begin{array}{c}\text { Inmigrantes de } \\
1^{\mathrm{a}} \text { generación }\end{array}$ & $\begin{array}{c}\text { Inmigrantes de } \\
1^{\mathrm{a}} \mathrm{y} \mathbf{2}^{\mathrm{a}} \text { generación }\end{array}$ \\
\hline España & 0,4529 & 0,4440 \\
\hline Alemania & 0,5651 & 0,3948 \\
\hline Austria & 0,4635 & 0,3893 \\
\hline Bélgica & 0,4778 & 0,4236 \\
\hline Bulgaria & 0,8489 & 0,7274 \\
\hline Croacia & 0,5737 & 0,3143 \\
\hline Dinamarca & 0,5407 & 0,4417 \\
\hline Eslovaquia & 0,8733 & 0,7537 \\
\hline Eslovenia & 0,5634 & 0,4506 \\
\hline Estonia & 0,8576 & 0,4925 \\
\hline Finlandia & 0,5565 & 0,4954 \\
\hline Francia & 0,5659 & 0,4477 \\
\hline Grecia & 0,5553 & 0,4081 \\
\hline Hungría & 0,7691 & 0,5646 \\
\hline Irlanda & 0,3358 & 0,3033 \\
\hline Italia & 0,5260 & 0,4188 \\
\hline Lituania & 0,9397 & 0,7859 \\
\hline Luxemburgo & 0,3052 & 0,1668 \\
\hline Malta & 0,5693 & 0,4589 \\
\hline Países Bajos & 0,6327 & 0,4756 \\
\hline Polonia & 0,9608 & 0,9398 \\
\hline Portugal & 0,5192 & 0,4332 \\
\hline Reino Unido & 0,4608 & 0,4703 \\
\hline Rep. Checa & 0,7408 & 0,5947 \\
\hline Rumanía & 0,9617 & 0,8970 \\
\hline Promecia & 0,4653 & 0,3988 \\
\hline Frodio Unión Europea & 0,6185 & 0,5035 \\
\hline
\end{tabular}

Fuente: Elaboración propia a partir de datos de PISA-2015 
es superior a países como Portugal $(0,43)$, Italia $(0,42)$, Bélgica $(0,42)$, Grecia $(0,41)$, Suecia $(0,40)$, Alemania $(0,39)$ o Irlanda $(0,30)$; pero sigue siendo inferior a otros como Francia $(0,45)$, Países Bajos $(0,48)$ o Finlandia $(0,50)$.

Esta nueva consideración del estudiante "no nativo", atendiendo no solo a su lugar de nacimiento sino también a sus orígenes culturales, muestra una situación considerablemente diferente a la planteada con anterioridad. Recordemos que, mientras en países como Reino Unido, Irlanda o Italia hay un mayor número de estudiantes inmigrantes de $2^{a}$ generación, en Alemania, Dinamarca o España la situación es la contraria. Sea como fuere la inclusión de los estudiantes inmigrantes de $2^{a}$ generación se plantea cada vez más como un problema que Europa debe afrontar.

En este caso, como decimos, el orden de los países se modifica de manera importante. En primer lugar, es relevante constatar que la segregación disminuye significativamente en todos los países al considerar también a los hijos de inmigrantes. Concretamente pasamos de un valor promedio de la UE de 0,62 con los inmigrantes de $1^{a}$ generación, a un valor promedio de 0,50 si se considera a los inmigrantes de $1^{a}$ y $2^{a}$ generación, lo que ya de por si es una buena noticia y genera optimismo en el futuro. De hecho, la segregación baja en todos los países excepto en Reino Unido, donde asciende ligeramente y da muestra de la problemática de integración que tiene ese país. La tendencia opuesta es

Gráfico 2. Segregación escolar por origen nacional en España comparada con los países de la Unión Europea. Índice de Gorard para los estudiantes inmigrantes de $1^{\mathrm{a}}$ generación y de $1^{\mathrm{a}}$ y $2^{\mathrm{a}}$ generación
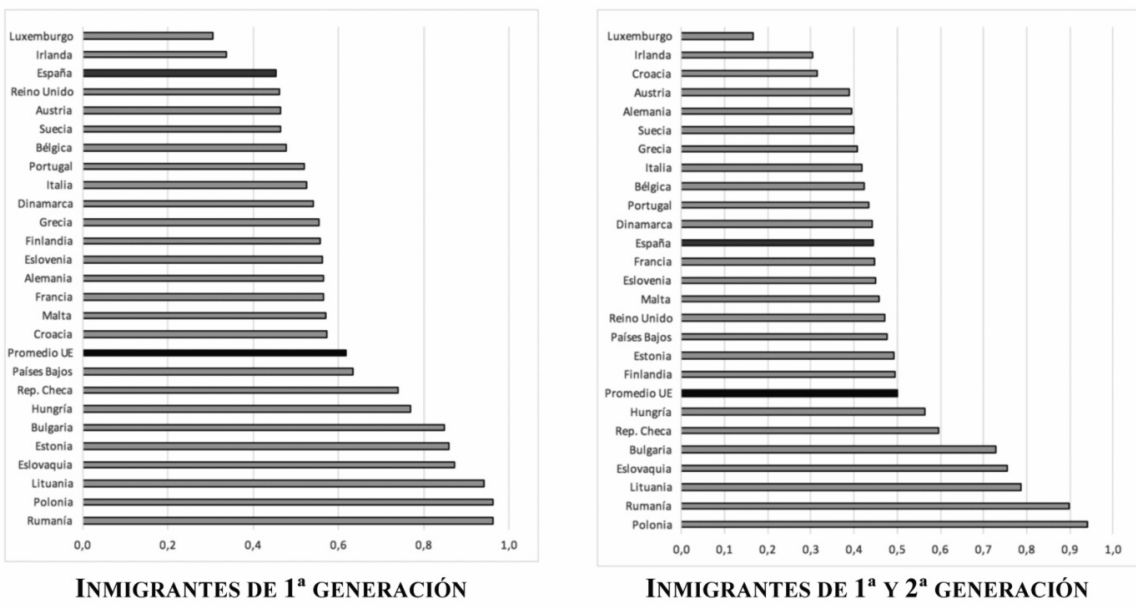

Fuente: Elaboración propia a partir de datos de PISA-2015 
liderada por Estonia (de 0,86 pasa a 0,49), Croacia (de 0,57 a 0,31), Hungría (de 0,77 a 0,56) y Alemania (de 0,56 a 0,39). Así, aunque Luxemburgo e Irlanda se siguen manteniendo como los países con menor segregación, le siguen Croacia, Austria y Alemania.

\section{Relación entre segregación y porcentaje de inmigrantes}

Una mirada más detallada sobre los anteriores resultados nos alerta sobre la estrecha relación entre el porcentaje de estudiantes inmigrantes escolarizados en una Comunidad o país y la segregación por origen nacional.

Efectivamente un simple cálculo del índice de correlación de Pearson entre el porcentaje de inmigrantes (tanto de $1^{a}$, como de $1^{a}$ y $2^{a}$ generación) y el índice de Gorard para análoga población nos indica:

- En el estudio de la segregación de las Comunidades de España: una correlación de $\mathrm{r}_{\mathrm{xy}}=-0,686$ (sig. 0,000) entre el porcentaje de inmigrantes de $1^{a}$ generación escolarizados y el índice de Gorard con inmigrantes de $1^{a}$ generación, y de $r_{x y}=-0,726$ (sig. 0,000) para los de $1^{a}$ y $2^{a}$ conjuntamente.

- En el estudio de la segregación de los países de la Unión Europea: una correlación de $\mathrm{r}_{\mathrm{xy}}=-0,598$ (sig. 0,001) para inmigrantes de $1^{\mathrm{a}}$ generación y de $r_{x y}=-0,675$ (sig. 0,000) para $1^{a}$ y $2^{a}$ generación.

Todas las relaciones son, por tanto, altas y negativas. Es decir, cuantos más estudiantes inmigrantes escolarizados hay en una Comunidad o un país, más bajo es el índice de segregación que posee. Ello nos lleva a pensar que, para ver las diferencias reales de segregación en cada Comunidad o país, sería necesario "descontar" el efecto debido al número de inmigrantes que hay en el mismo. Veámoslo por partes.

Comenzando por el estudio de la segregación en las Comunidades Autónomas de España, en el gráfico 3 se puede observar la relación entre el porcentaje de inmigrantes en una Comunidad y el índice de segregación por origen nacional. Allí se percibe que la posición de las diferentes Comunidades se ajusta con bastante precisión a una imaginaria línea decreciente (la línea de regresión de pendiente negativa), lo que muestra esta alta y negativa relación.

Verificada la relación entre el índice de segregación y el porcentaje de estudiantes, sería posible profundizar más en el conocimiento de la segregación "descontando" la influencia del número de inmigrantes escolarizados. Esta nueva perspectiva permite visualizar el esfuerzo real para la incorporación equitativa de los inmigrantes en las escuelas en cada Comunidad. En el gráfico 4 se presentan los residuales tipificados del análisis de regresión con el índice 
Gráfico 3. Relación entre porcentaje de estudiantes inmigrantes de $1^{a}$ generación en una Comunidad y el índice de segregación de Gorard

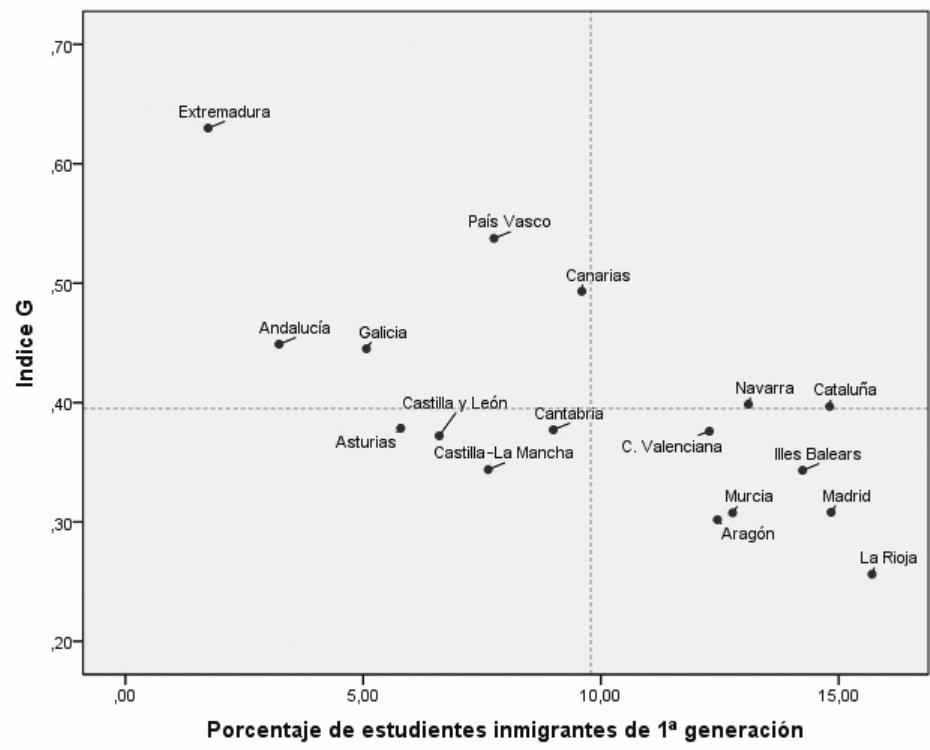

Fuente: Elaboración propia a partir de datos de PISA-2015

de segregación como variable dependiente y el porcentaje de inmigrantes en cada Comunidad como variable explicativa. Con ello se tiene una imagen de la segregación controlando el porcentaje de inmigrantes.

La panorámica que se obtiene es radicalmente diferente a la anterior. En este caso, hay cuatro comunidades que tiene una segregación mucho más alta de la que sería esperable: Cataluña, Canarias, País Vasco y Extremadura (residuales superiores a 1). Podemos suponer que en estas Comunidades las políticas de integración del alumnado inmigrante están siendo menos exitosas en comparación con, por ejemplo, las tres Comunidades que tienen una segregación mucho menor de lo previsible (residuos menores de -1): Castilla-La Mancha, Asturias y Castilla y León.

Análogos planteamientos podemos hacer para el estudio de la segregación por origen nacional en los países de la Unión Europea. En el gráfico 5 se muestra la relación entre el número de inmigrantes de $1^{a}$ generación escolarizados a los 15 años en cada país y el índice de segregación de Gorard para el mismo colectivo. De nuevo es posible observar una estrecha relación entre ambas variables. 
Gráfico 4. Segregación escolar por origen nacional en España controlando el número de inmigrantes de cada Comunidad Europea

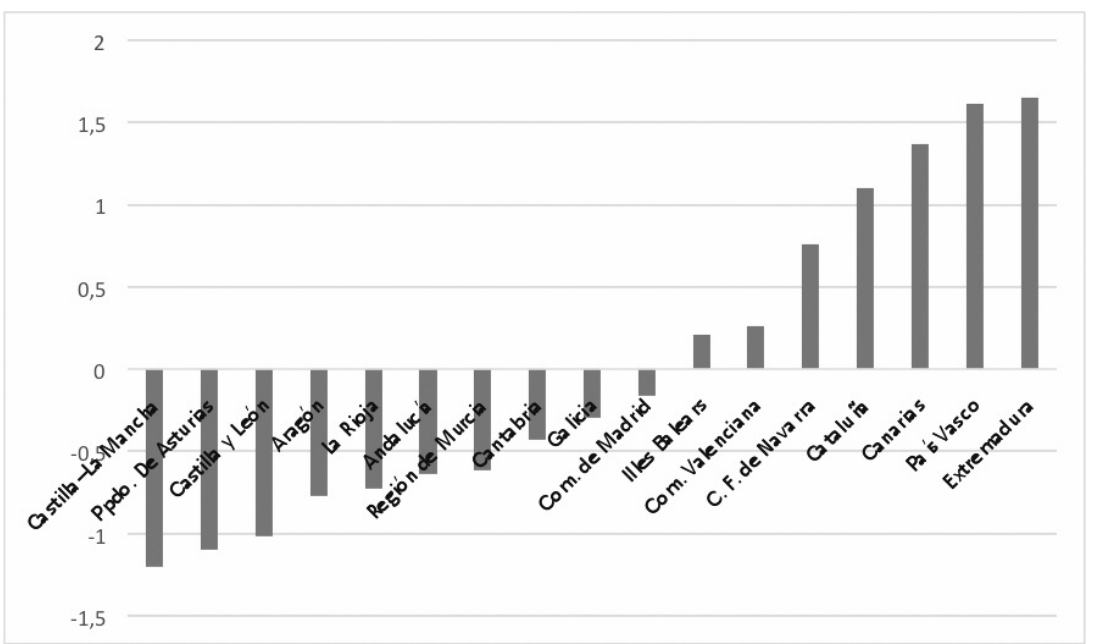

Fuente: Elaboración propia a partir de datos de PISA-2015

Gráfico 5. Relación entre porcentaje de estudiantes inmigrantes de $1^{\text {a }}$ generación en cada país de la Unión Europea y el índice de segregación de Gorard

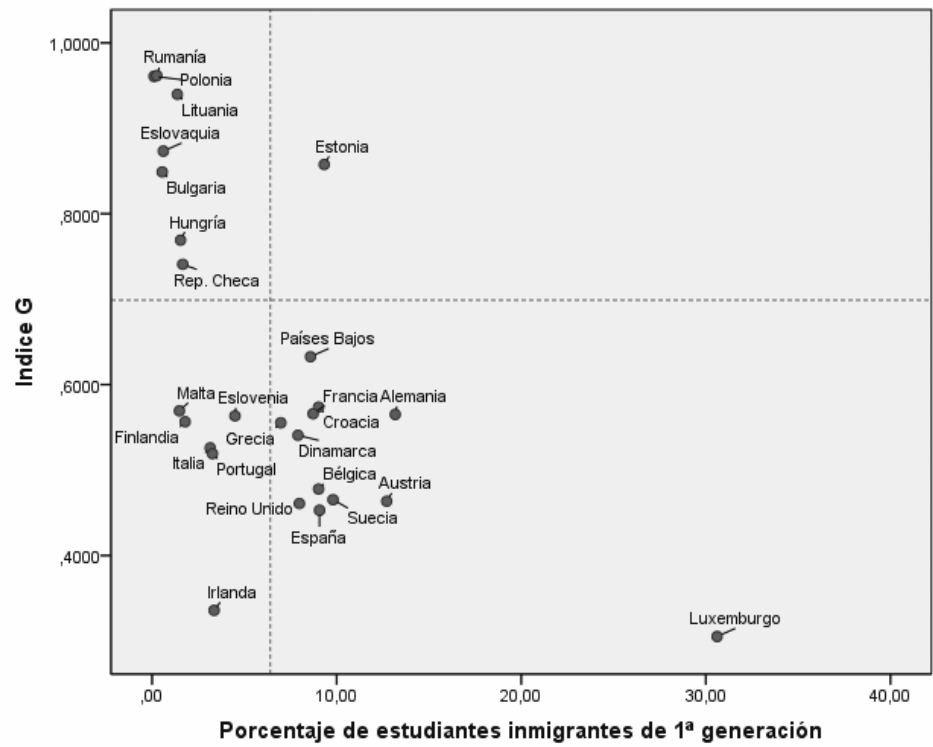

Fuente: Elaboración propia a partir de datos de PISA-2015 
Si, como hicimos con las Comunidades Autónomas, descontamos del índice de segregación la aportación del número de inmigrantes en cada país a través del cálculo de los residuales del análisis de regresión, encontramos interesantes resultados (gráfico 6).

Esta nueva imagen de la segregación neta nos muestra algunos cambios notables. Quizá el más llamativo sea el de Luxemburgo que ha pasado de ser el país con menor segregación bruta a uno de los que mayor tiene en términos relativos; situación análoga a la de Alemania que escala en puestos en segregación. En todo caso, exceptuando Eslovenia y Países bajos, el gráfico nos muestra que siguen siendo los países del este europeo los que más segregan, tanto en términos brutos como netos. Y los países de la Europa occidental los países que menos segregan. Concretamente los que menos segregan son Irlanda, Italia, Portugal y Finlandia.

Esta nueva visión de la segregación neta ofrece una imagen menos optimista para España. De ser el tercer país con menos segregación, hemos pasado a ser el séptimo; con más segregación que Reino Unido, aunque aún por debajo de otros como Bélgica, Suecia, Grecia o Francia.

\section{Gráfico 6. Segregación escolar por origen nacional en los países de la Unión Europea controlando el efecto del número de inmigrantes de cada país}

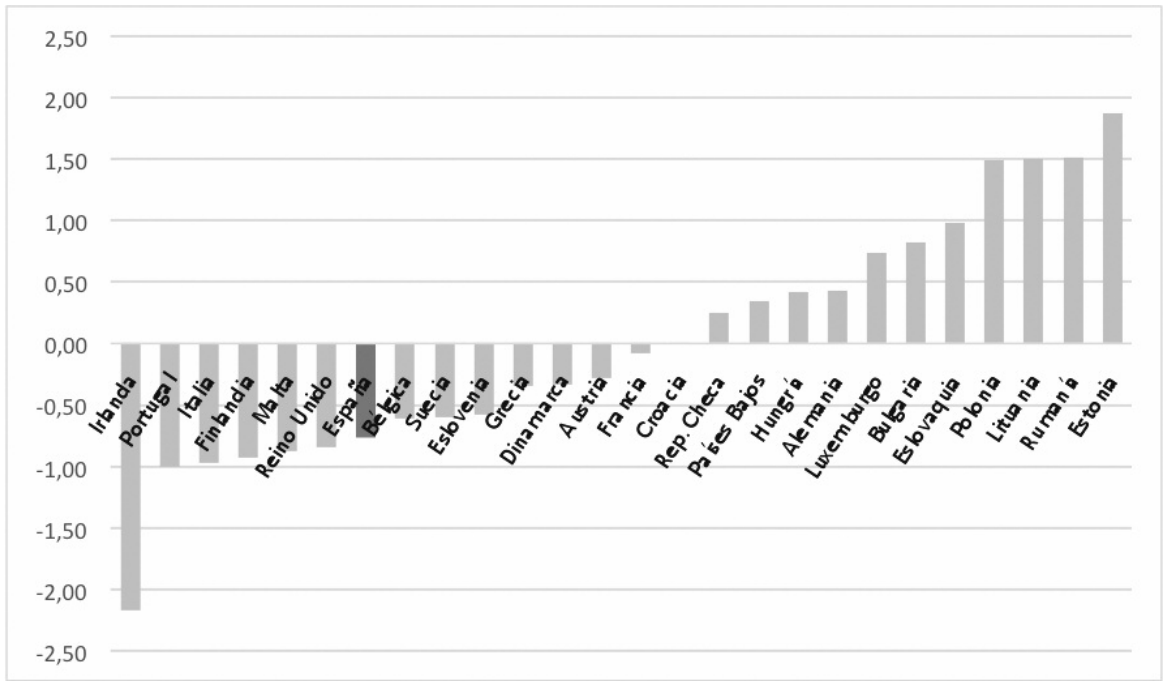

Fuente: Elaboración propia a partir de datos de PISA-2015 


\section{DISCUSIÓN Y CONCLUSIONES}

Esta investigación ha demostrado, con datos actuales y estadísticamente representativos, que la segregación escolar por origen nacional es un grave problema en España. Si queremos una sociedad más justa e inclusiva es imprescindible un sistema educativo de análogas características, pero la existencia de una segregación de 0,45 de magnitud medido con el índice de Gorard es una muestra de que estamos muy lejos de tenerlo. Esta idea coindice plenamente con trabajos anteriores realizados con distintos índices. Recordemos, por ejemplo, que Sánchez Hugalde (2007) encontró valores de segregación de 0,62 en la ciudad de Barcelona con el índice de Disimilitud; para Cataluña, Bonal (2012) estimó una segregación de 0,19 medido con el índice de Hutchens; o 0,46 en 2006 en Cataluña, medido con el índice de Disimilitud (Valiente, 2008). Recordamos también que, según estos trabajos, la segregación es mayor en Secundaria y mayor entre los inmigrantes de $1^{a}$ generación que entre los de $2^{a}$ generación (como también aquí hemos demostrado).

También se ha encontrado que la segregación en la gran mayoría de los países de la Unión Europea es superior, o incluso muy superior que la de España, especialmente si se consideran solo los inmigrantes de $1^{a}$ generación. Estas cifras altas de la segregación en Europa ya fueron halladas por otros trabajos como los de Dronkers y Levels (2008), Entorf y Lauk (2008) o Schnepf (2006), entre otros.

Uno de los hallazgos más interesantes encontrados es la relación entre segregación escolar por origen nacional y el porcentaje de inmigrantes en el sistema educativo. Este hecho, sin embargo, ya fue detectado por Schnepf (2006) y nos pone sobre aviso en uno de los factores que inciden en la segregación. Pero no es el único. Como bien señalaron Bonal y colaboradores (2005), o más recientemente Espejo Villar (2011) para el caso de España, la gestión y la dirección de la educación y sus consecuencias en forma de segregación escolar por origen nacional responden a los diferentes procesos de descentralización seguidos y la orientación ideológica de cada gobierno autonómico.

De esta forma, este trabajo es pionero en visibilizar el esfuerzo de los gobiernos de cada Comunidad por generar políticas de desagregación escolar. Los datos son persistentes en afirmar que Cataluña, Canarias, País Vasco y Extremadura tienen mucho recorrido por hacer.

La legislación española es rotunda en prohibir cualquier tipo de discriminación y de selección basada en el origen nacional de los estudiantes para todos los centros financiados con fondos públicos (que implica la inmensa mayoría de los centros en España). Sin embargo, resulta esclarecedor diferenciar el plano entre lo que es y lo que debería ser (nuestros datos apuntan que el valor pro- 
medio de la segregación escolar por origen nacional en España es 0,45, en las Comunidades Autónomas esta cifra puede variar desde 0,26 en La Rioja, hasta 0,60 en Extremadura). En los años 50 del siglo pasado, Estados Unidos declaró inconstitucional la separación de estudiantes en función de su raza en distintas escuelas, alegando que atentaba al principio de igualdad de oportunidades educativas. La cifra de 0,45 de segregación escolar por origen nacional en España hace pensar si una discriminación prohibida de iure, se hace efectiva en los hechos. La desigual distribución de los estudiantes según sus características personales atenta contra la convivencia en pluralidad. Resulta paradójico que mientras que la globalización y el desarrollo tecnológico generan las bases materiales para difuminar los límites y las distancias entre naciones, los muros de las escuelas separan a los adolescentes.

Sabemos que disminuir la segregación no garantiza necesariamente un trato justo y equitativo para todos los estudiantes, pues el mero hecho de compartir centros educativos no excluye problemas como el asimilacionismo, el no reconocimiento, la discriminación o la exclusión. Estas cuestiones, obviamente, no pueden ser verificadas en este estudio. Sin embargo, se puede deducir fácilmente que el hecho de no compartir establecimiento ni siquiera deja la posibilidad de mejorar la convivencia dentro del centro escolar. El estudio deja en evidencia que la situación es problemática, pues, el origen de una construcción intercultural es compartir el espacio escolar, situación que para casi la mitad de adolescentes españoles inmigrantes no se está dando (Aparicio y Portes, 2014).

Este estudio presenta algunas aportaciones, pero también limitaciones. Entre las primeras hemos de destacar el trabajar con datos actuales y fiables, y una muestra representativa para cada Comunidad Autónoma. La base de datos PISA-2015 aporta informaciones que no se disponían hasta el momento. No olvidemos que se analizan datos de una muestra significativa de 39.066 estudiantes escolarizados en 1.177 centros educativos españoles a los que hay que añadir, 164.938 estudiantes y más de 6.000 escuelas en la Unión Europea. Sin embargo, la muestra no es significativa al nivel de centros y el contar con pocos estudiantes por escuela podría ocultar segregación entre aulas, así como otro tipo de segregaciones intra-escolares.

La investigación sobre segregación escolar en España es joven -recordemos que sus inicios datan de principios del 2000, y que incluso, la gran mayoría de los estudios se realizaron en la Comunidad Autónoma de Cataluña-. Los resultados de la investigación realizada se suman a los hallazgos de las investigaciones previas reforzando la necesidad de que los investigadores aborden el estudio de la segregación en las escuelas del país. Por ejemplo, investiga- 
ciones sobre la aportación de la educación pública y privada, el estudio diferencial de los inmigrantes con familias de nivel socioeconómico más bajo, o los efectos y las causas de la segregación. Por supuesto, una aproximación cualitativa a la segregación escolar ayudará también a comprender mejor el fenómeno y sus consecuencias. Con sus resultados, se darán pasos hacia el desarrollo de políticas educativas que hagan frente a la situación de segregación en el país.

No es posible construir una sociedad justa y equitativa con sistemas educativos que segregan a los estudiantes por su lugar de nacimiento o por el de sus padres. Esta investigación ha aportado datos que demuestran que la segregación escolar por origen nacional es un problema en España que exige medidas urgentes. Aunque en el contexto de la Unión Europea las cifras de España podrían resultar quizá bajas, especialmente en lo referido a los inmigrantes de $1^{a}$ generación, la estimación de la aportación neta de cada país no ofrece cifras $\tan$ optimistas.

Si y solo si conocemos un fenómeno, podremos tomar medidas eficaces para combatirlo. La segregación escolar es, hoy por hoy, un grave problema en España. Parece claro que la legislación por sí sola no es capaz de acotar el fenómeno de la segregación escolar por origen. Urgen medidas, por tanto, que hagan que esa legislación se cumpla y que de verdad construyamos un sistema educativo más inclusivo que contribuya a transformar la sociedad para que sea más justa. Ello no solo es posible, es necesario.

\section{REFERENCIAS}

Aja, E. (2000). La regulación de la educación de los inmigrantes. En E. Aja, F. Carbonell, Colectivo Ioé, J. Funes e I. Vila (Eds.), La inmigración extranjera en España. Los retos educativos (pp. 69-98). Barcelona: Fundación La Caixa.

Alegre, M. À., Benito, R. y Gonzàlez-Baetbó, I. (2008). Procesos de segregación y polarización escolar: La incidencia de las políticas de zonificación escolar. Profesorado, Revista de Currículum y Formación del Profesorado, 12(2), art 8.

Aparicio, R. y Portes, A. (2014). Crecer en España. La integración de los hijos de inmigrantes. Barcelona: La Caixa.

Benito, R. y González-Baetbó, I. (2007). Processos de segregació escolar a Catalunya. Barcelona: Mediterrània.

Bonal, X. (2012). Municipis contra la segregació escolar. Sis experiències de política educativa local. Barcelona: Fundació Jaume Bofill.

Bonal, X., Rambla, X., Calderón, E. y Pros, N. (2005). La descentralización educativa en España. Barcelona: Fundació Carles Pi y Sunyer.

Burgess, S., McConnell, B., Propper, C. y Wilson, D. (2004). Sorting and choice in English secondary schools, Working Paper 4/111. Bristol: Centre for Market and Public Organisation. 
Capellán de Toro, L., García, J. F., Olmos A. y Rubio, M. (2013). Sobre agrupamiento, concentración, segregación o guetización escolar: Claves para un análisis interpretativo de tales situaciones y procesos. Avances en Supervisión Educativa, 18, 1-27.

Dronkers, J. y Levels, M. (2008). Do school segregation and school resources explain region-of-origin differences in the mathematics achievement of immigrant students? Educational Research and Evaluation, 13(5), 435-462.

Duncan, O. y Duncan, B. (1955) A methodological analysis of segregation indexes. American Sociological Review, 20, 210-217.

El-Habib, B., Jiménez-Delgado, M., Ruiz Callado, R. y Jareño Ruiz, D. (2016). Composición escolar y expectativas del alumnado por origen y etnia: una aproximación a la segregación escolar extraoficial. RASE: Revista de la Asociación de Sociología de la Educación, 9(1), 59-77.

Entorf, H. y Lauk, M. (2008). Peer effects, social multipliers and migrants at school: An international comparison. Journal of Ethnic and Migration Studies, 34, 633654. https//doi.org/10.2139/ssrn.908844

Espejo Villar, B. (2008). El fenómeno de la inmigración en España y en las políticas educativas territoriales. Revista Española de Educación Comparada, 14, 13-47

Frankenberg, E. (2013). The role of residential segregation in contemporary school segregation. Education and Urban Society, 45(5), 548-570.

Gallego, R. y Subirats, J. (Dirs.). (2011). Autonomies i desigualtats a Espanya Indice Igualdad. Cataluña: IEA.

García Castaño, F. J. y Olmos Alcaraz, A. (2012). Segregaciones y construcción de la diferencia en la escuela. Madrid: Editorial Trotta.

Garreta, J. (2011). Las experiencias escolares de la inmigración. Papers, 96(1), 205-223.

Gorard, S. y Taylor, C. (2003). What is segregation? A comparison of measures in terms of 'strong' and 'weak' compositional invariance. Sociology, 36(4), 875-895. https//doi.org/10.1177/003803850203600405

MECD. (2016). Estadísticas de la Educación en España. Madrid: Ministerio de Educación Cultura y Deporte.

Murillo, F. J. (2016). Midiendo la segregación escolar en América Latina. Un análisis metodológico utilizando el TERCE. REICE. Revista Iberoamericana sobre Calidad, Eficacia y Cambio en Educación, 14(4), 33-60. https//doi.org/10.15366/reice2016.14. 4.002

Murillo, FJ. y Martínez-Garrido, C. (2018). Magnitud de la Segregación escolar por nivel socioeconómico en España y sus Comunidades Autónomas y comparación con los países de la Unión Europea. RASE: Revista de la Asociación de Sociología de la Educación, en prensa.

Noguera, P. A., Pierce, J. C. y Ahram, R. (2015). Race, equity, and education. Nueva York, NY: Springer.

OCDE. (2016). Programme of international student assessment (PISA 2015). París: OCDE. Palaiologou, N. (2007). School adjustment difficulties of immigrant children in Greece. Intercultural Education, 18(2), 99-110. https//doi.org/10.1080/14675980 701327205 
Sánchez Hugalde, A. (2007). La segregació escolar dels immigrants a Catalunya. Quaderns d'Avaluació, 13, 55-76.

Schneeweis, N. (2011). Educational institutions and the integration of migrants. Journal of Population Economics, 24(4), 1281-1308. https//doi.org/10.1007/s00148009-0271-6

Schnepf, S. V. (2006). How different are immigrants? A cross-country and cross-survey analysis of educational achievement. En C. Parsons y T. M. Smeeding (Eds.), Immigration and the transformation of Europe (pp. 245-276). Cambridge: Cambridge University Press.

Schnepf, S. V. (2007). Immigrants' educational disadvantage: an examination across ten countries and three surveys. Journal of Population Economics, 20(3), 527-545.

Simpson, L. (2007). Ghettos of the mind: The empirical behavior of indices of segregation and diversity. Journal of the Royal Statistical Society, 170(2), 405-424. https//doi.org/10.1111/j.1467-985x.2007.00465.x

Sindic. (2008). La segregación escolar en Cataluña. Barcelona: SINDIC

Stroub, K. J. y Richards, M. P. (2013). From resegregation to reintegration: Trends in the racial/ethnic segregation of metropolitan public schools, 1993 2009. American Educational Research Journal, 50(3), 497 531. https//doi.org/10.3102/00028312 13478462

Terren, E. y Carrasco, C. (2007). Faith in school: Immigrant families' attitudes towards education in Spain. International Studies in Sociology of Education, 17(4), 389-406. https//doi.org/10.1080/09620210701667061

Valiente, O. (2008). ¿A qué juega la concertada? La segregación escolar del alumnado inmigrante en Cataluña (2001-06). Profesorado. Revista de Currículum y Formación del Profesorado, 12(2), 1-23.

F. JAVIER MURILLO. Profesor titular del Área de Métodos de Investigación y Diagnostico en Educación de la Universidad Autónoma de Madrid. Coordinador del grupo de investigación "Cambio Educativo para la Justicia Social" y Secretario Académico del Instituto de Derechos Humanos, Democracia, y Cultura de Paz y No Violencia (DEMOSPAZ) de la UAM. Página web de docencia: http://uam.es/javier.murillo. ORCID ID: 0000-0002-8003-4133. Email: javier.murillo@uam.es

CYNTHIA MARTÍNEZ-GARRIDO. Profesora Ayudante Doctor del Área de Métodos de Investigación y Diagnóstico en Educación de la Universidad Autónoma de Madrid. Doctora en Educación por la Universidad Autónoma de Madrid. Miembro del Grupo de Investigación "Cambio Educativo para la Justicia Social (GICE)". Página web: www.cmartinezgarrido.es ORCID ID: 0000-0001-7586-0628. 
GUILLERMINA BELAVI. Es Licenciada en Ciencia Política (orientación Análisis Político) por la Universidad Nacional de Rosario (Argentina). Actualmente realiza sus estudios de doctorado gracias a un contrato pre-doctoral de Formación del Personal Investigador (FPI-UAM). Es miembro del grupo de investigación "Cambio Educativo para la Justicia Social" (GICE), su área de especialización es la educación democrática. ORCID ID: 0000-0002-6471-7583.

Recibido: 03/02/2017

Aceptado: 28/07/2017

(c) (P) Licencia Creative Commons Reconocimiento (CC BY 4.0) 